qui alimentait le moteur synchrone sans faire décrocher celui-ci. On a terminé l'essai en supprimant le courant alternatif qui alimente le moteur synchrone, tout en laissant en pleine charge le redresseur-régulateur. Celui-ci se met hors phase et s'arrête progressivement. Le

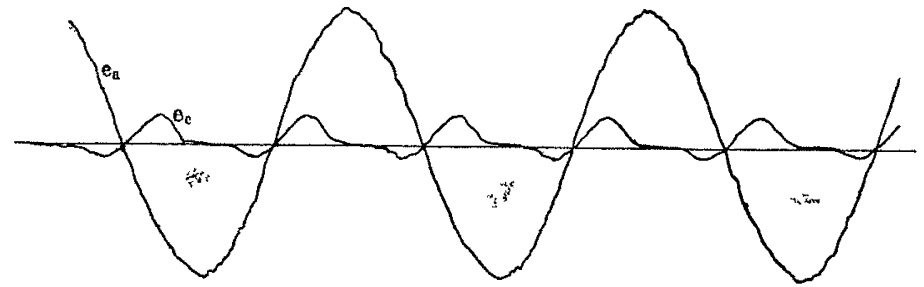

Fig. 4. - Les balais mobrles sont au commencement de la course, dans la position la plus rapprocliée des balas tixes. $e$ a tension alternatire, lof volts elficaces,

$e_{\mathrm{c}}$ teusion totale moyenne redressée, 22 volts.

courant redressé est remplacé par du courant allernatif dont l'intensité est forcément très réduite par la forte selfinduction du circuit. D'allleurs il ne se produit rien d'anormal sur les collecteurs qui continuent à fonclionner sans étincelles.

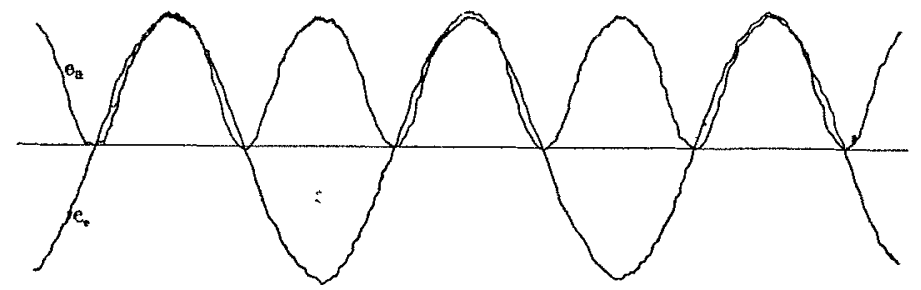

Fig. 5. - Les balass molıles sonl à fond de course.

ea tension alternative, 167 rolts efficaces, ec tension totale moyenne 230 rols.

Un avantage particulier du redresseur-régulateur réside dans ce fait qu'il se prête avec la plus grande facilité à la récupération de l'énergie, car cet appareil est entièrement réversible. Et si l'on emploie sur les locomotives des moteurs à excitation séparée, on pourra maintenir sur les déclivités telle vitesse que l'on voudra, sans faire usage des freins, et en restituant à la ligne l'énergie qui serait inutilement absorbée par les sabots des freins. Enfin, il serait possible de faire usage de batteries tampons, comme on le fait pour la traction par courant continu (*).

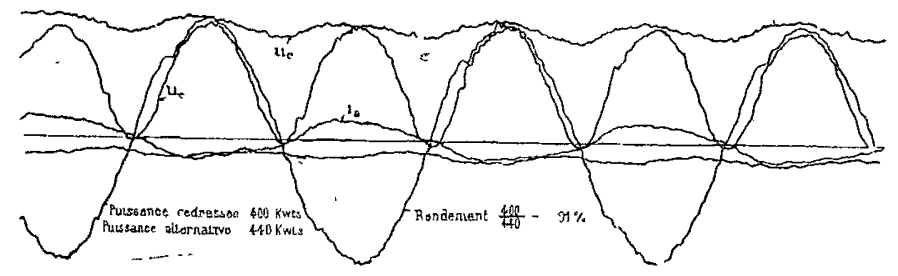

Fig 6. - Les balais mobiles sont à fond de course dans la posilion la plus éloıgnée des balais fixes.

$U_{\mathrm{a}}=155$ volts efficaces $U_{\mathrm{c}}=235$ volts moyen.

$i_{\mathrm{a}}=1390 \mathrm{amp}$. efficaces $i_{\mathrm{c}}=1500 \mathrm{amp}$. moyen.

Un calcul détaillé, fait en vue de l'application de la traction électrique sur la ligne d'Alais à Langogne, a montré qu'en employant le régulateur-redresseur on pourrait, grâce à la récupération, diminuer de 27 pour 100 la quantité d'énergie électrique. En tout cas, on conçoit sans peine qu'on réaliserait une économie en employant cet appareil sur les lignes à profil très accidenté $\left.{ }^{* *}\right)$.

(*) Ces batteries seraient, bien entendu, placées dans la station centrale, ou dans des sous-stations appropriées.

(“) Lors de sa communication au Congrès de Lyon, M. Auvert a déclaré que, à la suite d'un concours, son redresseur-régulateur avait été adopté pour l'alimentation des locomotives qui feront le service sous le tunnel du Loechesberg dont le percement vient d'ètre décidé.

\section{LE MOIS HYDRO-ÉLECTRIQUE}

\section{ACADÉMIE DES SCIENCES}

\section{MÉCANIQUE ET ÉLECTRICITÉ}

Résistance des électrolytes pour les courants de haute fréquence. - Note de MM. André Broca et S. Turchini, séance du 28 mai r 906 .

La théorie de Lord Kelvin relatıve à la résistance des conducteurs cylindriques pour les courants de haute fréquence condurt, dans le cas des métaux, à des résultats qui présentent avec l'expérience des différences systématiques, comme nous l'avons montré 1 l y a un an (Comples rendus, t. CXL, p. I 228). Nous avons repris ces expériences en nous adressant aux électro!ytes, pour lesquels la théorie doit s'appliquer comme pour les métaux, espérant trouver des divergences analogues. La difficulté était seulement de rédiser un conducteur électrolytique assez gros pour que les effets de concentration à la surface pussent s'y produire, malgré la faible conductibilité de ces corps. Nous avons opéré sur un cylındre de $6 \mathrm{~cm}$ de diamètre et de $10 \mathrm{~cm}$ de long, et nous avons eu des résultats nets en employant l'eau acıdulée, à partir d'une concentration suffisante.

Nous avons commencé par vérifier, au moyen de notre électrodynamomètre précédemment décrit, qu'un ampèremètre à fil chaud spé cialement construit pour les courants de haute fréquence donnail des indications exactes dans les limites de période entre lesquelles nous avons opéré ; cela a rendu les détermınations ultérieures beaucoup plus faciles. Les perturbations dues a la tréquence deviennent négligeables dans le cas de fils aussı fins que ceux quı sont employés dans ces appareils.

L.e princıpe de la méthode est le même que dans notre étude des fils métalliques. On mesure d'une part l'intensité efficace du courant de haute fréquence, et d'autre part l'échauffement qu'il produst dans le conducteur électrolytique, en le traversant pendant une minute. On recommence l'éxpérience en mesurant l'échauffement produit dans ce même conducteur par un courant alternatif à 42 périodes passant pendant le mème temps et ayant la même intensité efficace. Le rapport des deux échauffements donne le rapport des résistances du conducteur pour le courant de haute fréquence et pour le courant alternatıf ordinaire. Nous admettons que, pour la très basse fréquence de celui-ci, la résistance est la même qu'en courant continu, et nous pouvons, par son emploi, nous mettre à l'abri des phéno. mènes délectrolyse.

Pour mesurer l'echauffement dû au passage du courant, le tube qui contient le liquide est munı d'un tube capillaire latéral, dans lequel on mesure lascension du liquide échauffé. Le courant est amené dans l'appareil par deux larges électrodes de platune de $6 \mathrm{~cm}$ de dramètre. 'Tout l'appareil est soigneusement enveloppé de coton pour le mettre à l'abri des courants d'air. On commence toujours par lire pendant quelques minutes la marche normale de réchauffement ou de refroldissement de l'appareil, et on opère quand cette marche est bien constante et ne dépasse pas le vingtrème de l'échauffement attendu.

On peut voir que, dans les circonstances où nous sommes, les effets observés sont dus uniquement à l'échauffement, et que les modifications chimiques n'y entrent pour rien. Nous avons en effet des électrodes absolument symétriques, et toutes les réactions réversibles par nature sont éliminées par le fait même. S'll s'en passe qui ne le soient pas, elles ne peuvent non plus avorr d'action sensible. $\mathrm{M}$. Rothé a montré en effet que les électrodes prenaient, au bout d'un très petit nombre de cycles de potentiel, un état permanent qui correspond à un cycle de polarisation toujours identique à luimêne. D'allleurs, quand la dilution devient assez grande, le fast que les deux espèces de courant donnent la même chose, semble bren prouver qu'il en est ainsi. De plus, dans le cas du sulfate de cuivre, les résultats sont les mêmes avec des électrodes en cuivre et avec des electrodes en platine.

Nous avons ainsi obtenu le résultat suivant, en appelant $R_{f}$ la résistance en haute fréquence et $R_{c}$ la résistance à basse fréquence, supposée égale à celle qui existe en courant contınu.

Eau acidulée très étendue de sulfate de cuivre $\frac{R_{f}}{R_{c}}=\mathrm{I}$

\begin{tabular}{|c|c|c|}
\hline $\begin{array}{l}\text { Eau acidulée } \\
\qquad \frac{1}{10}\end{array}$ & & $\begin{array}{l}\text { Enu actudulée } \\
\text { au manumum } \\
\text { de conductublitit }\end{array}$ \\
\hline Fréquence. & $\frac{R_{i}}{R_{c}}$ & Fréquence \\
\hline 3000000 & 0,77 & 3000000 \\
\hline 2000000 & 0,9 & $10000 r^{\prime} 0$ \\
\hline 500000 & 0,8 & $900 \quad 000$ \\
\hline 190000 & 0,8 & $\begin{array}{l}500000 \\
1900(0\end{array}$ \\
\hline
\end{tabular}

En somme, nous arrivons à ce résultat surprenant : 
Quand la conductibilité est suffisante pour permettre l'ohservation d'un changement de résistance d'un électrolyte, l'échauffement de celui-ci est moindre avec le courant de haute fréquence qu'avec le courant de basse fréquence de mẹme intensite efticace, contrairement à ce que la théorie semble faire prévoir.

Asclépiadées nouvelles de Madagascar produisant du caoutchouc. - Note de MM. J. Constanrin et I. Galtaud. Séance du 25 juin 1906.

M Geay, voyageur du Muséum, qui parcourt en ce moment Madagascar, nous a adressé, entre autres documents, des échantillons complets de tiges, feulles, fleurs et fruits, secs $\in t$ conservés dans lalcool, appartenant à deux Asclépiadées nouvelles dont le latex foumit du caoutchouc $\left(^{\star}\right)$. Ces deux plantes, très abondantes dans les planes alluviales de l'ouest et du sud-ouest de Madagascar, oú elles sont distınguées sous les noms de Komptsé et de Dangolora, sont deux hanes sarmenteuses d'exploitation facile d'où les indigènes retient du cáoutchouc. Ce produit soumis à l'appréciation de techniciens, notamment de $M$. Michelin, a été reconnu " utulisable et de bonne qualité moyenne ». Il est donc uttle de faire connaître ces plantes qui, jusqu'à présent, n'ont pas encore été sıgnalées, et ciur poutront piendre de limportance dans l'exploitation de notre colonie.

Kompitsé. - C'est une liane sarmenteuse, formant souvent buisson, appartenant au groupe des Périplocées; elle constitue un genre nouveau et une espece nouvelle pour laquelle nous proposons le nom de Kompitsia elastica:

Feuilles opposées, à limbe quelquefois arrondi dans les feuilles de hase des rameaux, mais le plus souvent étroit, allongé. de $7 \mathrm{~cm}$ de long sur $\mathrm{r} \mathrm{cm}$ de large en moyenne, aıgu au sommet et rétrécı en com à la base sur un court petivle de quelques millimetres.

Inflorescences terminales ou axillares, en cymes bipares, régulieres, bien fournies. Calice vert à 5 dents étroltes, recourbees en dehors vers la base de la fleur. Corolle rose à 5 dents étroites, allongées, de couleur plus vive que le tube, tordues dans le bouton, recouvrant à droite et formant aınsi un bec allongé. Les dents de la corolle étalee ont $\mathrm{r} \mathrm{cm}$ de long, $3 \mathrm{~mm}$ de largeur médiane et surmontent un tube corollare court, présentant 5 renflements ovalatres correspondant aux dents. Coronule simple, formée de 5 dents triangulatres, sallantes à la gorge et s'insérant dans les echancrures de la corolle sur une base élargie par deux oreillettes laterales Filets staminaux soudés à la corolle depuis la base et ne s'en détachant que sur une farble longueur. Anthères allongées, pourvues de deux aurıcules à la base. Pollen en tétrades. Translateurs sans rétinacle, en forme de gouttier e allongée avec partie terminale d'abord étranglée puis étalée en pelle. Pistil caché dans le tube sous les dents de la coronule; style renfle en massue, pourvu de 5 satlles entre lesquelles sont pincés les translateurs; tête stıgmatique conique courte. Fruit formé de 2 carpelles non soudes, dans le prolongement l'un de l'autre à maturité complète, à surface glabre, pourvu de côte's longitudinales; dimensions d'une carpelle: $70 \mathrm{~mm}$ sur $5 \mathrm{~mm}$ Graines à aigrette caduque, couvertes de tins tubercules brun racao et présentant un sillon longitudinal; dans le fruit elles sont rangées en deux séries séparées par une lame parcheminée $\left({ }^{*}\right)$.

Un certain nombre de ces caractères sont ceux des genres Cryptolepis, Stomatostemma et Raphasme. Toutefois, dans le Kompitse, le calice, le tube de la corolle et surtout la coronule sont de forme différente, ce quı nous parait justıfier la création d'un genre nouveau pour cette plante. Elle donne un latex abondant renfermant du caoutchouc, mas d'ordinaire les indigènes le mélangent au latex de l'autre Asclépiadée, le Dangolora, que nous décrirons prochannement et qui est voisıne du genre Marsdena. Le mélange du latex des deux plantes donne le caoutchouc connu à Madagascar sous le nom de Manomby.

\section{GEOLOGIE ET HYDROLOGIE}

Sur la rapidité de l'érosion torrentielle. Note de M. E.-A. Martel, séance du i 8 juin igo6.

J'ai noté dernièrement (Comptes rendus, 5 mars 1906) que le grand cañon du Verdon. paraît être très jeunc et que son approfondissement se poursuit encore rapidement $\left({ }^{\star \star *}\right)$

Depuis longtemps, une autre localıté m'avait frappé quant aux changements manifestes qui s'y sont produits au cours de quelques

(") On ne connaît à Madagascar que trois Asclépiadées donnant du caoutchouc. Ce sont: le Cryptostegra madagascarensis, le Maisdenta cencosa et un Secamone (vahm.untr) signale par M. Jumelle (Journal du caoutchonc et de la gutta-percha, 15 jullet 1905.

Kopitso Muséum vient de recevoir de $M$ de Vilmorin, sous le nom de pante à des graines dépourvues d'aigrette qui ressemblent de facon frapété mises graines de Kompitsé, lorsque l'argiette est tombee. Ellles ont tité.

**) Voir La Houlle Blanche, juin rgo6. années sculement, c'est la perte et la rivière souterraine de Bramadiau (Gard) que j'al décrite 1 ci même (Comptes rendus 4 décem. bre is88). Depurs 1884, je m'y surs rendu dix fois et dès 1807 , avec mon collaborateur M. F. Mazaurıc, j'avais remarqué des altération fort nettes en divers points, principalement à la sortie de la caverne, par où réapparaît le cours d'eau.

Ma dernère visite ( 28 mal igo6) m’a montré que, depuis igoo, en six annécs, ll est encore survenu des modifications vrament profondes.

A la partie si péricure, l'issue du tunnel de la Baume (depuis longtcmps abandonné par le torrent) a contunué de se démolir si activement, par suite de l'infiltration des eaux fluviales, qu'on ne peut plus approcher du bord du précipice sur lequel il débouchait.

A la sortie de Bramabiau, un éboulement a cmporté le sentier quı accédait à la grande diaclasc par où la rıvıè̀e reçoit le jour ; dans cette diaclase, les crues souterraines ont dégradé les strates forman corniches naturelles cl'accès (sur $200 \mathrm{~m}$. de potondeur), où l'on avalt posé en I 899 desi mains-courantes et gardes-fous en fer, qui n'exıstent déjà plus , enfin la grande cascade par où le Bramabiau effectue son septième it dernicr bond souterrain nest plus reconnaissable, si l'on compare les photographics de i $88+$ et même de 1899 avec ccllcs de 1906. Il y a vingt-dcux ans, cette chute s'épanchait en arc de cercle par-dessus une sallie du rocher formant tablette convexe ; maintenant, et à volume ógal d'eau, clle est logée tout entère dans unc rigole en gouttière, profondément excavée sur la rive droite de la sail lie, tablette dont tout le surplus est à sec La cascade large tend à se transformer en rapide étroit.

Tout ceci n'a rien de particulier en cc qui touche les effets bien connus de l'usure des rochcrs par érosion mécanıque : mais le poin de vue nouveau prut-êtrc, c'cst que la friabilité ct la fissuration cxtrême des calcarres bruns de l'infra-lias à Bramabiau permet à ce cffets de se produire avec une rapidité très grande et gui n'est pas plus exceptionnelle que celle des deux classiques excmples du Simeto (Sicılc) et du Niagara Il importe en cffet de la mettre on parallèlo avec ce qu'on a observé, en ces demières années, aux endrolts suivants

$I^{\circ}$ Marmites de la Maigrange à Fribourg (Sunse) creusécs en 25 ans (1872-1897) dans la mollasse (/. Brunhes)

$2^{\circ}$ Cañon de $9^{8 \mathrm{~m}}$ de long sur $1^{\mathrm{m}} 3 ;$ à $3^{\mathrm{m}} 7$ de profondeur, creusé en 36 heures par un orage et une crue (It-1 5 avril igoo) dans les marne et la dolomie de la rivière Schlocke à Schmarden, près Rıga ( $B$ Dosz).

$3^{\circ}$ Enlèvement d'un énorme rocher ou pont naturel de Ponadieu (Alpes-Maritımes) par l'orage du 16 octobre rss6 (A Guébhard).

$4^{\circ}$ Perte de plus en plus sensible du Danube à Immendingen (Quenstedt, Endriss, Penck).

$5^{\circ}$ Approfondisscment de cent mètıts, evécuté de 1850 à 1890 , par le torient de Rovana (Tessin) au pied de la terrasse de Campo (prof. Herm)

$6^{\circ}$ Elargissement des fissures amont du goufre de Gaping-Ghyll (Yorkshire) qui, de 1805 à 1903 , a mis presquà sec (par érosion régressive) le puits principal de l'abîmc où s'engloutit le ruisscau de FellBeck (Cuttriss)

$7^{\circ}$ Déblaiements réalisés, par les crues de 1903, dans les couloirs souterrains de l'abîme d'Eastwater Swallet, près Wells, Somerset (M. Balch), etc.

Il serait facile de multiplier ces cxemples. Ceux-ci suffisent pour imposer la conclusion suivante.

S'il est des cas et des roches dures et homogènes, où les effets de l'érosion demeurent pratiquement non enregistrables par les mesures de temps humaines, il y par contre nombre de sites, où la fissilité et l'inconsistance de la prerıe permettraient de constater matériellement ces effets au cours même de quelques années.

Par conséquent, ct comme on le fait depurs longtemps pour la recherche des crues et décrues des glaciers, 11 importeralt d'établir, par des moyens faciles à organiser, ct là où les conditions lithologiques et hydrauliques s'y prêterarent le micux, des stations d'observations photographiques et autres, smon permanentes, du moins périodiques pour les altérations érosives des cours d'eau. Il est superflu d'insister sur les précieuses indications que de pareilles constatatrons fourniraicnt pour la meilleure utılisation des torıents, des rivières torrentielles et de la fameuse houlle blanche, ainsi que pour la sauvegarde de certains travaux publics ; en maintes circonstances notamment, on pourrait ainsi prévolr, éviter, corriger les conséquences de changements de biefs, déplacements de cours, obstructions de lits, destructions d'accès, transformations de débits, disparitions de barrages naiurels, etc Bref, ce serait un appoint de plus, et précisément daos les points particulièrement préjudiciables par la rapidité de leurs altérations, pour le plus complet asservisscment économique de l'cau, cette rebelle ct toute pussante force, contre laquelle la lutte industrielle et hygiénique doit se poursuivre de plus cn plus opmiâtrément.

Nous rappelons que tout ce qui concerne la Rédaction doit etre adressé au rédacteur en chef, M. Cote, 24, rue Sully, à LYON, et que tout ce qui concerne l'Administration doit être adressé aux éditeurs, MM. Gratier et Rey, Grande Rue, à Grenoble. 


\section{INVENTIONS NOUVELLES}

Procédé et dispositif pour extraire les métaux des minerais et autres matières métallifères, par dissolution et électrolyse. Brevet no 356.825 , Socı́́x́́ Ganz, I I août i 905 .

La présente invention est relative à un procédé et à un dispositıf rour la dissolution des métaux, au moyen de radıcaux acides qui prennent naissance dans l'électrolyse des sels alcalins, et en particulier des chlorures: l'électrolyte étant, à la cathode, separé par un diaphragme du restant du liquide, afin que les produits de l'electrolyse qui se déposent à la cathode purssent être séparément éloignés. Le nouveau procédé se distingue des procédés connus en ce sens que le courant n'est pas conduit à travers la matıère à lessiver; l'espace où l'électrolyse est pratiquée est disposé aul-dessous de l'espace qui sert au lessivage, et il est séparé de ce dernier par un fond perméable, ce qui permet aux cathodes et aux anodes d'être disposées dans un voisinage immédiat les unes des autres. Dans ces conditions, 11 ne se produit dans la chan-bre d'électrolyse une decomposition de l'électrolyte qu'en tant que les cations (l'alcali dans l'espèce) qui se forment à l'intérieur des diaphragmes sont séparés du bain, alors que les anions, c'est-à-dire le radical acide, sont, par le liquide de l'anode, entraînés de bas en haut, vers la chambre de lixiviation et dissolvent le métal en traversant la couche de matières à lessiver. Les électrodes sont régulièrement réparties sous le fond qui porte la couche de mınerai, et cela en grand nombre et de manière à ne pas gêner l'écoulement de l'électrolyte de bas en haut. Dans l'execution du procédé, il importe particultèrement que l'électrolyte qui circule passe tout d'abord devant les anodes et ensuite seulement
L'auge servant à l'électrolyse est construite en une matière imperméable résistant aux acides, par exemple en béton, ou en bois im. prégné d'asphalte ou d'autre matière analogue, en maçonnerie, etc.

Dans la forme d'exécution représentée, le fond C est en béton; les parois latérales D sont en briques cuites avec des ancrages en fer tra. versant la maçonnerie et reliant des cadres supérieur et inférleur avec lesquels ils forme une armature robuste augmentant la résisstance des parois minces contre toute pression latérale. Un faux-fond E-E, perforé d'orifices $a$, de préférence horizontal, et qui, sur le dessin est représenté en forme de voûte, sépare l'auge en deux cham. bres, l'une supérieure, l'autre inférieure.

La chambre F, au-dessous du fond perforé $E$, forme la chambre d'électrolyse ou de décomposition, tandis que la chambre supéi ieure $G$ forme la chambre de lixiviation ou de dissolution où l'on verse la matière à lessiver $\mathrm{H}$.

Dans la chambre de décomposition $F$, sont disposés les diaphragmes $d$, qui peuvent être avantageusement de forme tubulaire, placés hor zontalement ou inclinés, et faits en une matière résistant aux alcalis, telle que le ciment, l'amiante, le parchemin, un tissu de coton nitré. A l'intérieur des diaphragmes tubulaires se trouvent les chambres à cathodes qui contiennent les cathodes décrites ci-après.

Les diaphragmes tubulaires passent isolement à travers le paroide la cuve, aux extrémités qui renferment les bornes d'attache pour les cathodes, et sont en ce point pourvus de têtes à joint étanche g, qui font saillie au dehors et sont individuellement reliées, par des raccords $m$, au collecteur d'alcali I. Chaque tube-diaphragme peut ainsi être séparément examiné et changé au besoin. Les autres extrémités des tubes diaphragmes sont réunies en groupes par le moyen de tubes en $\mathrm{T} f$, pour diminuer les points de passage à travers la paroi. La formation de gtoupes est pourtant nécessaire, car il ne seratt pas avantageux d'alimenter tous les tubes-diaphragmes par un conduit

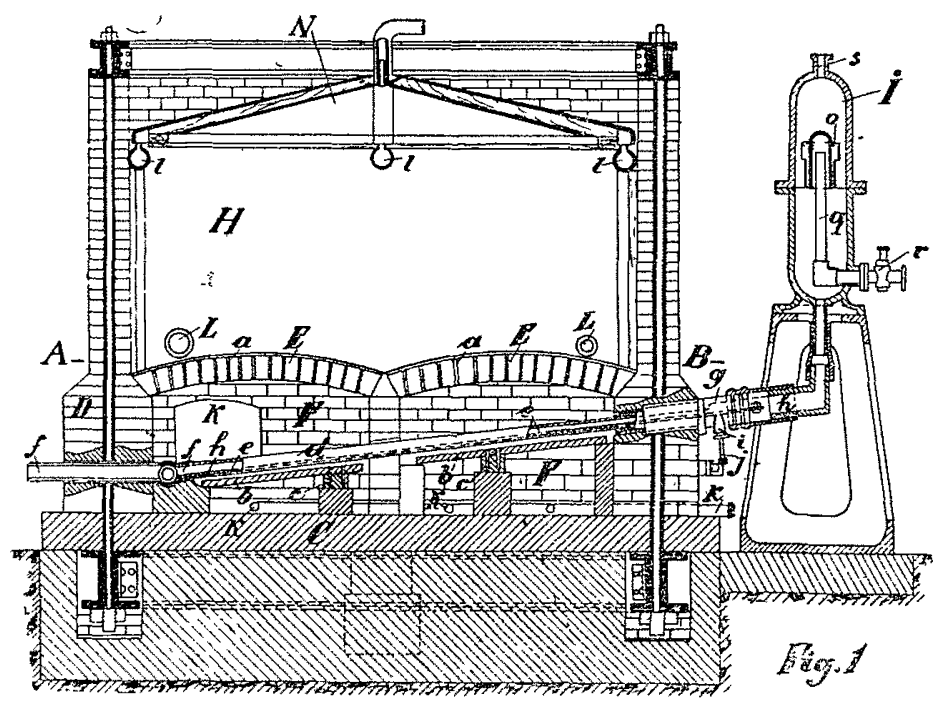

devant les diaphragmes qui contiennent les cathodes, de facon que l'électrolyte soit, avant d'atteindre les draphragmes, assez saturé déjà des anıons acides pour que la lessive filtrant à travers ces derniers ne précipite pas, sous forme d'hydrates ou d'oxychlorures, les métaux de l'électiolyte qui, pendant le cycle précédent, a absorbé des sels métalliques.

Les avantages du procédé consistent en ce que la résistance de la cellule (vase poreux) est considéi ablement réduite, vu que les électrodes peuvent être disposies dans une ćtrolte proximité l'une de l'autre et que le courant n'a pas à traverser la couche de minerais. D'autre pait, les anions produits sont utilisés aussı complètement que possible, parce que d'un côté la résistance de la couche de minerai n'entre pas en considération, et que l'importance de la couche peut en conséquence être augmentée suivant les besoins, et que, d'un autre côté, le chlore, qui éventue!lement n'auraıt pas été absorbé, peut se dégager librement et être uulisé, par exemple, pour la production du chlorure de chaux, d'acide chlorydrique, etc. De plus, la formation, dans la cellule, de précipités métalliques basıques est évitée. Enfin, gràce à la séparation entre la chambre de lixiviation et la chambre d'électrolyse, l'introduction des matieres à traiter et la séparation des résidus peuvent s'effectuer sans déranger en rien le dispositıf d'élec1rolyse; en outre, toute avarie des électrodes et des diaphragtnes, Eausce par les minerais, se trouve exclue.

L.e dessin ci-joint représente, à tıtre d'exemple, une forme d'exécution du dispositif pour la mise en pratique du procédé

La fig. I est une coupe verticale transversale du dispositif;

La fig. 2 est une élévation en bout;

Les fig. 3 et 4 représentent, à plus petite échelle, des élévations latérales, respectivement du côté où est anıené le liquide de la cathode ct de celui ou s'écoule l'hydarate alcalin formé;

La fig. 5 est une coupe horizontale, à plus petite échelle, suivant $A-B$ de la fig. I.

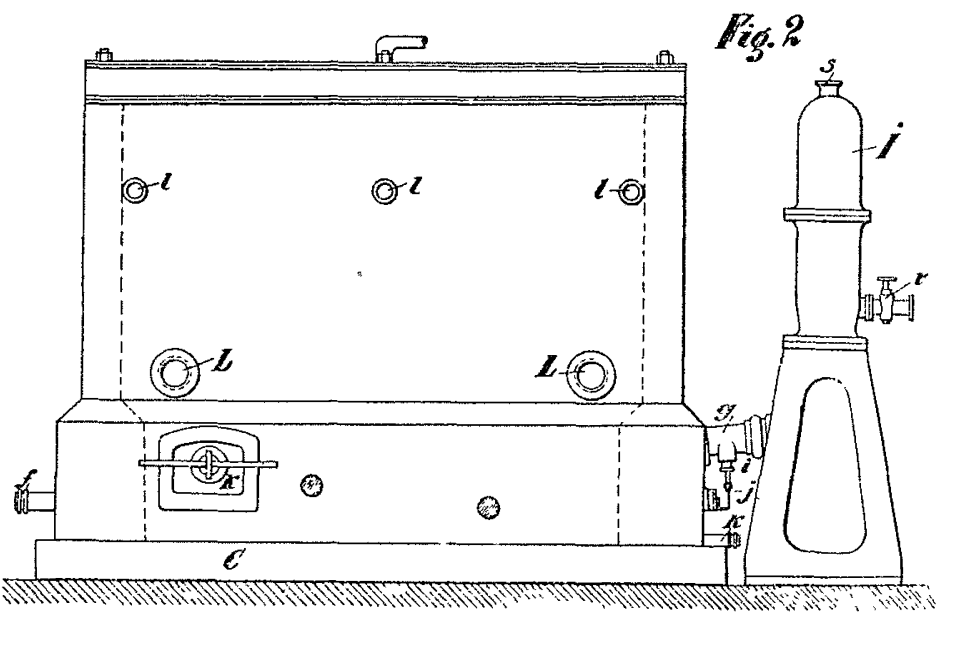

d'alimentation commun $f$, parce qu'il est important que l'écoulement du liquide à travers les diverses chambres à cathodes se fasse aussi uniformément que possible. Les conduits d'alimentation $f$, ainsi que les têtes $g$, doivent être en une matière résistant aux alcalis, de préférence en faience. Les tubes-diaphragmes sont, au moment du montage, d'abord cimentés dans les têtes $g$ puis mis en place dans la cure, après quoi les extrémités inférieures, accessibles par un trou d'homme $k$, sont reliées et cimentées aux tubes d'alimentation.

Dans la chambre de décomposition $F$, on dispose au-dessous des diaphragmes, ou entre eux, mais jamais par dessus, les anodes $b$ de forme quelconque appropriée, en une matière insoluble, graphite ou charbon par exemple, et auxquelles le courant est amené parles conduits d'alimentation $c$ faits de la même manière et passant avec jolnt étanche à travers la paroi de l'appareil.

La fig. 5 montre les huit premiers tubes-diaphragmes de gauche réunis en un groupe par un conduit d'alımentation $f$; à côté l'on v'olt douze plaques d'anodes. Les anodes du premier groupe, disposées sous les tubes diaphragmes ainsi que les diaphragmes qui se trouvent au-dessus des anodes représentées, ont, pour plus de clarté, été supposés enlevés.

L'importance que cette disposition présente pour le pı océdé réside en ce fait que, si l'on dissout par exemple du cuivre par le chlore, l'électrolyte ancdique affluant de bas en haut touche d'abordles anodes et y prend du chlore en quantité suffisante pour qu'en passant devant les diaphragmes, à travers lesquels filtre toujours un peu d'alcal, il ne puisse précipiter des oxychlorures ou des hydrates de cuivre de l'électrolyte déjà chargé de cuivre.

Les cathodes $h$, qui pourront avantageusement être en fils ol rubans de nickel ou de fer, sont disposées dans les tubes diaphrames. Le courant est amené par les tıges métaillıques $j$, de préterence en cuivre, emmanchées étroitement dans les branchements-j des têtes $g$, et reliées au rail collecteur $M$. 
La chambre de dissolution $\mathrm{G}$ est remplie du minerai à traiter $\mathrm{H}$ qui repose sur le fond perforé E. Dans la partie supérieure de la quimbre de dissolution sont disposés des tubes $l$ qui servent à l'écoulement de la solution de sel métallique obtenue. Les tubulures qui débouchent dans la partie inférıeure de la chambre de disso. lution, servent au rinçage des résidus.

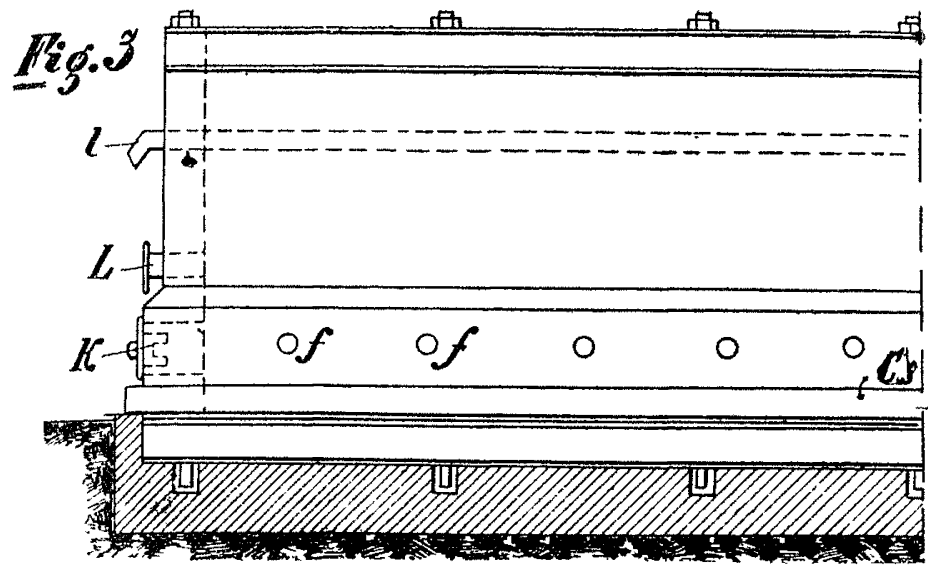

La fig. I montre un couvercle $\mathrm{N}$ dont les bords s'appliquant exactement aux parois de l'auge plongent dans le liquide et forment ainsi une fermeture étanche au gaz. Le couvercle peut être soulevé au moyen de chaìnes ou d'autres organes et sert à recueillır ou à séparer les radicaux acides gazeux non utilisés, le chlore par exemple. Le fond perforé $E$ est recouvert d'une couche perméable filtrante, lésistant aux acides, d'une toile d'amiante par exemple, pour éviter la chute des poussières de minerais dans la chambre d'électrolyse ou l'obturation des orifices du faux fond $E$.

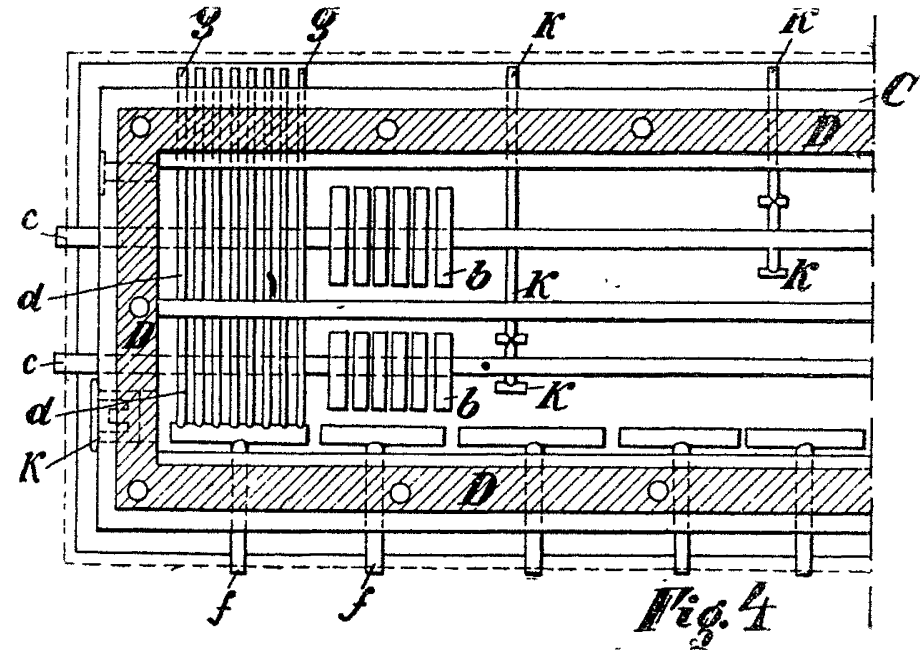

Le mode de fonctionnement du dispositif est le suivant :

Le lıquide anodique qui au début de l'opératıon consıste erı une solution de sel alcalin, mais plus tard en une mélange de cette solutıon saline et de solution de sel métallique à obtenır, pénètre par les tubes $k$ (i.g. 5) en matière résistant aux acides, sous les anodes, dans la chambre de décomposition $\mathrm{F}$.

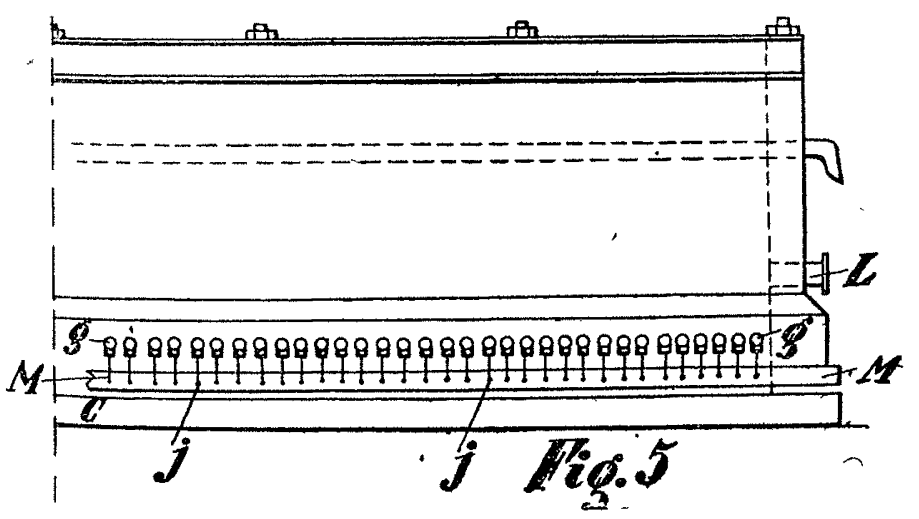

Tandis que l'électrolyte affluant de bas en haut passe devant les anodes, il recuetlle les radicaux acides produits, par exemple le chlore, et les entraîne, par-dessus les orifices $a$ du fond séparateur $E$, dans la chambre de dissolution $G ; 1$ l traverse uniformément la couche de minerai $\mathrm{H}$, dissout les métaux qu'elle contient et sort de l'appareil par les tubes $l$. Recueillie, la solution est, suivant les conditions de l'exploitation, immédiatement démétallisée pour être ramenée ensuite dans la chambre d'électrolyse de l'apnareil, ou bien l'électrolyte, aı ant sa demétallisation, est conduit à plusieurs reprises à travers l'apparell jusqu'à ce qu'll ait atteint la teneur en sel voulue.

La solution de sel alcalin qui forme la liqueur cathodique arrive, par les tubes d'alimentation $f$, dans les chambrts à cathodes $e$, c'està-dire dans les tubes-diaphragmes, où le métal alcalin est séparé des cathodes $h$ et, comme on sait, forme immédiatement avec l'cau un hydrate alcalın. Le mélange de solution de sel alcalın et d'hydrate alcalın et d'hydrogène aınsı produit arrive, par les têtes $g$ et les raccords $m$, dans le vase collecteur I, séparé de l'appareil de dissolution et avantageusement construit en fer. Dans ce vase I l'hydrogène s'accumule dans la chambre à gaz $n$, tandis que le liquide débarassé du gaz s'écoule dans la chambre $o$. La chambre $o$ est formée à l'extrémite supérieure d'un conduit d'échappement de gaz $q$ pourvu d'un séparateur du gaz $p$, de facon que le niveau du liquide s'y trouve être à peu près le même que celuı de l'électrolyte anodique dans la chambre de dissolution, c'est-à-dire à la hauteur des conduits de décharge $l$.

Tandis que le mélange de la solution d'hydrate et de sel alcalin s'écoule par le conduit de decharge $q$ et le robinet de fermeture $r$ solt pour recommencer un nouveau cycle, solt pour être dirigé immédiatement vers le dispositıf de la chambre à gaz, séparateur de sel l'hydrogène s'echappe par l'orifice $s$ pour être recueilli et utilısé à volonté. Quand le minerar est suftsamment épuisé, on soutire la liqueur anodique de la cuve par les tubes $k$ et la liqueur cathodique par les tubes $f$, puis les iésidus sont lavés et chasses de la chambre de lessivage à travers les tubulures $L$. Les dichets sont, de la faonç connue, amenés au crassier.

RÉsumé : Io Un procédé pour extraire les métaux des minerais et autres matières métalliferes par vole électrolytıque au mojen d'un électrolyte en circulation développant des anions dissolvants, les liqueurs anodique et cathodique etant séparées par des diaphragmes, et caractérisé en ce que l'électrolyte est électrolyse dans une chambie située au-dessous de la couche de matière à traiter, et séparée de cette dernière par un fond perforé da façon que le liyuide aftluant ne peut qu'après dvoir touche les anodes, lécher les daphragmes renfermant la liqueur cathodique et que le liquide anodıque chargé des anions dissolvants traverse en s'elevant ld couche à lessiver, dans le but d'utiliser parfaitement les anions produits par un renforcement correspondant de la couche de mineiais et une réduction aussi grande que possible de la résıstance de la cellule et d'éviter la formation de précıpités métalliques basiques sur les diaphragmes.

$2^{\circ}$ Un dispositif pour l'exécution de ce procédé caractérisé en ce que la cuve est séparée, par un faux-fond perforé, en une chambre supérieure pour la matière à traiter et une chambre inférieure servant à l'électrolyse et dans laquelle les électrodes des deux pôles sont régulièrement répartıes sous le faux-fond et aussı rapprochées que possible les unes des autres, de facon que l'électrolyte afluant lèche d'abord les anodes puis les draphragmes contenant les cathodes.

3o Une forme d'exécution dans laquelle les diaphragmes tubulaires sont disposés au-dessus des plaques d'anodies (qui recouvrent tout le fond de la cuve en laissant des intervalles pour le passage de l'électrolyte) de façon à former une grille recouvrant toute la surface du tond et à travers laquelle la liqueur anodique peut couler.

$4^{\circ}$ Une forme d'exécution dans laquelle les diaphragmes tubulaires sont, par une extrémité, isolément engagés du dehors à travers la paroi de la cuve, au moyen de têtes cimentées qui renferment des bornes de prise de courant et de façon queles têtes passent au dehors et peuvent isolément ètre relıées aú collecteur d'alcalı.

Procédé et dispositif pour extraire les métaux des minerais et autres matières métallifères, par dissolution et électrolyse. Addition au précédent Brevet, 12 août I905, n 5 . 106.

Dans l'exécution pratique du procédé décrit dans la demande du I I août, il est important que les tubes-diaphragmes puissent facilement, et aves un joint étanche, être engagés dans l'appareil, et que la cathode, qui doit être disposée à l'nntérieur du tube-diaphragme puisse aussi y être introduıte avec facilité et en être extraite de mème sans empêcher, par sa présence, le démontage aisé de la tubulure de raccord cntre les tubes diaphragmes et le collecteur d'alcali.

La prise de coúrant, représentée en coupe longitudınale sur le dessin c1-joint, répond à ces desiderata multiples.

La tête $b$ porte les anneaux en caoutchouc $c$ qui servent à assurer l'étanchéité entre cette tête de tube et l'oritice de la paroi de l'auge à électrolyse; elle porte, à une extrémité, une tubulure plus étrolto a dans laquelle peut être emmanché le tube-diaphragme enveloppé d'une matière appropriée pour assurer l'etanchéité à l'eau. L'autre extrémité de la tête porte la tubulure élargie $d$, munie d'une tubulure latérale $e$. Le bord $f$ de la tubulure $d$ sert à recevoir la piece de i accord qui assure la liaison avec le récipient du collecteur d'alcali. Par la tubulure d'embranchement $e$ est engagéc une tige $i$, en cuivro par exemple, à l'extrémité intérieure de laquelle est vissée, rivée, soudée ou fixée de toute autre façon la borne d'attache pour la ca thode. Cette borne $j$ sera avantageusement en nickel ou en fer; elle est fixée de manière à faire saillie, par son extrémité, hors de la tu- 
bulure $d$ tout en restant à l'intérieur de la section droite du tube et facilitant d'une part une attache commode de la cathode et d'autre part une liaison sans encombre avec le collecteur d'alcalı.

La tıge de prise de courant $i$ est étanchérée dans la tubulure $e$, au moyen des rondelles en caouichouc $h$ comprimées entre la borne $j$ et l'écrou $g$ vissé sur ladıte tige $i$. L.e joint pourıait se farre difléremment, par exemple par un remplissage avec une natière appropriée.

Ains que le montre le dessin, la prise de courant $i$ forme un tout solide avec la tête a dans laquelle elle est fixée une fors pour toutes. Après que le tube-diaphragme a eté monté dans la parol de la cuve,

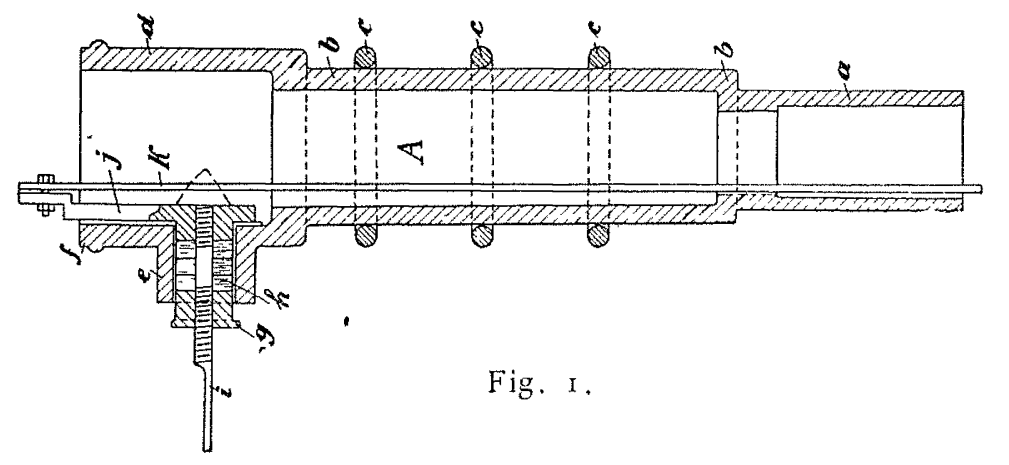

la cathode $k$ est poussée en place dans le tube, et l'extrúmıté de ladite rathode est relıce par des vis et soudée à l'extı émıté $\mathrm{d} z$ borne $j$ qui falt sallie à travers le tube.

RÉsumé - Pour le dispositif de la demande du I I août, une prise de courant pour des électrodes disposées dans des tubes isolants, caractérisée en ce qu'une pièce de prise de courant coudée (engagée avec joint étanche par la paroi d'une tubulure en matière résistante aux acides et aux alcalıs, et emmanchée sur le tube-diaphragme) dort l'extrémité qui tait sallie latéralement à travers la tubulure est reliee à la conduite de distribution, est aménagée de façon que la prise de jonction qui se trouve à l'intérıeur de la tubulure, fart saillıe pardessus son bord assez lon pour pouvorr étre reliee à l'extrémite des électrodes engagées dans le tube, sans prêfudıce pour la lıaison commode entre le tube et le collecteur d'alcali et le démontage facile de cette liaison.

\section{INFORMATIONS DIVERSES}

\section{Comité d'Electricité}

Par décret en date du 20 aoùt 1906 , rendu sur la proposition du ministre des travaux publics, des postes et des télégraphes, le comité d'électricité, dont la création a été prévue par l'article 20 de la loi du 15 juin r 906 sur les distributions d'énergie, a été composé ainsi qu'il suit :

Représentants professionnels français des grandes industries électriques : MM. Boutan, directeur de la Compagnie du gaz de Lyon ; Brachet, directeur du secteur électrique des ChampsElysets ; Brylinski, sous-directeur de la société du Triphasé ; Cordier, directeur général de la société « Energie électrique du littor al méditérranéen », président du Syndicat des forces hydrauliques: EQver, directeur de la Compagnie générale paris enne de tramways; Fontalne (Hippolyte), ingénieur électricien, administrateur des ateliers des machines Gramme; Gulllain, président du Consell d'administration de la compagnie tran. çaise pour l'exploitation des procédés Thomson-Houston; Harlé, de la maison Sautter-Harlé et Cie ; Hillairet, ingénieur-construteur, président de la Société des Ingénieurs de France; Lauriol, ingénieur en chef des services généraux d'éclairage de la ville de Paris; Mascart, membre de l'Insiitut, dire:teur du bureau central météorologique; MEYER(Ferdinand), d recteur de la compagnie continentale Edison; $P_{A v i E}$ directeur général de la Compagnie générale française de tramways; Picou, ingénieur des Arts et Manufactures; Sartiaux (Albert), ingénieur en chef de l'exploitation à la compagnie du chemin de fer du Nord.

Fonctionnaires, représentant des administrations publiques intéressées: MM. Bruman, conseiller d'Etat, directeur de l'administration départementale et communale; Collomb, chef du

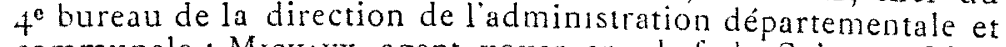
communale; Michaux, agent voyer en chef de Seine-et-Oise, - pour l'Administration de l'intérieur.

$\mathrm{MM}$. Maurice LÉvy, inspecteur général des Ponts et Chaus- sées de $1^{\text {re }}$ classe, membre de l'Institut; MONMERQué, ingénieur des Ponts et chaussées; Werss, ingénieur ordinaire des mines, - pour l'Adminisiration des travaux publics.

MM. Guillebot de Nerville, ingénieur des Postes et Télé. graphes, professeur adjoint d'électricité à l'école nationale des Ponts et Chaussées ; Maureau, ingénieur en chef drs Télégraphes; Devaux-Charbonnel, ingenieur des Télégraphes, pơlir l'Administration des Postes et Télégraphes.

$M$. le lieutenant-colonel Cunmer, chef de la section technique du génie; le lieutenant-colonel Bertrand, directeur du laboratoire des recherches relatives à l'aérostation militaire; le capitaine Cordier, professeur adjoint du cours d'artillerie ì l'école d'application de l'arillerie et du génie, - pour l'Admi. nistration de la guerre.

MM. Dabat, directeur de l'hydraulique et des améliorations agricoles; POCHET, inspecteur géréral de l'hydraulique agricole: IROTÉ, chef du service technique hydraulique, - pour l'Admi. nistrationde l'agriculture.

M. Mascart, a été nommé président du comité, et M. MonmerQué, a été nommé secrétaire.

\section{École Supérieure d'Electricité}

Ont obtenu le diplôme d'ingénieur-électricien les éléves sortants de la promotion 1 905-1 006: M M. de Pistoye, Regrault, Cany, Pésez, Rion, Rolland, Nicolas, Baudis, Gaucharaud, Ferron, Carentène, Cill't, Dervillé, Kostko, Chabot, Manescau, Jouffret, Vellutini, Pertuisot, Marcandier, Vautier, Schombourger, Gajan, Engelhard, Panthier, Roche, Jégou, Neufeld, Josse, Guinant, Gillequin, Hélitas, Comte, Mével, Fabre, Bloch, Durand, Laurhier, Polack, Frohlich. Miramont, Rossignol, Stugocki, C athiard, Bérenger, Déchandol, Legoupil, Puppati, Jacobsfeld, Borloz, Mounier, Jacobsen, Mathiell, Touchard, Lasserre, Barraud, Gatin, Gomien, Vincent (G.), Chapelle, Berger, Jean-Joseph.

Et les vétérans: $\mathrm{M}$ M. Sureau, Varlanesco, Letrou, Kienast.

Ainsi que les officiers suivants désignés par le Ministère de la guerre pour suivre les cours de l'Ecole : MM. les capitaines Viry et de Zienkiewicz; le lieutenant Roussel.

\section{Transmission d'énergie électrique dans le pays de Galles.}

Ce projet est le premier exécuté sur une grande échelle pour la transmission en Angleterre de l'énergie électrique obtenue avec des chutes d'eau.

L'eau vient des lacs Llyn, Lydaw et Glaslyn, qui se trouvent au pied du Snowdon, près du sentier que suivent los excursionnistes qui font à pied l'ascension de celte rnontagne.

La conduite d'eau débouche à la partie la plus basse de ces deux lacs, à $9 \mathrm{~m}$. au-dessous du niveau naturel de l'eau, au moyen d'un long tunnel entaillé dans le rocher. L'eau arrive ensuite à l'extrémité superieure de la conduite forcée, contistuée par des tuyaux en tôles d'acier rivées, de $7,1 \mathrm{~mm}$. d'épaisseur et de $76 \mathrm{~cm}$. de diamètre. A l'extrémité inferieure de la conduite d'eau, comme la pression augmente, on a accru un peu l'épaisseur et on a dıminué un peu le diamètre. La longueur moyenne des tuyaux est de $5 \mathrm{~m}$. 8o et on a employé 450 sections entre les lacs et l'usine génératrice, la conduite ayant une lon. gueur de $2 \mathrm{~km}$. 6 . Au dessus de la station, et à la partie supé. ricure du tuyau d'amenée, on a placé des joints flexibles et des soupapes d'air. La chute d'eau dont on dispose est de $360 \mathrm{~m}$. La station centrale a une longueur de $76 \mathrm{~m}$. et une largeur de $14 \mathrm{~m}$. arec une hauteur totale de $15 \mathrm{~m}$. 5 .

Les moteurs hydrauliques consistent en quatre roues Pelion attelées directement aux alternateurs triphasés de BrucePeebles, du type à inducteurs tournants, pouvant fournit chacun ' 1500 K.V.A.à 10000 volts, à 50 périodes par seconde, lorsqu'ils tournent à une vitesse angulaire de 500 cours par minute. On a laissé la place pour plusieurs roues alternateurs et en prévision d'une augmentation de la puissance demandée.

La ligne de transmission, qui doit transporter du courant 2 10000 volts, va de la station dans une direction ouest, vels Llanberis, et dans une direction sud-ouest, vers les carrières de Pakley.

Les câbles sont portés par des isolateurs maintenus au moyen 
de ters galvanisés posés sur les poteaux. Les tiges en fer galvanisé sont scellées dans les isolateurs au moyen d'un mélange de litharge et de glycérine. Les tiges en ter des isolateurs sont toutes placées du même côté du poteau, et elles sont à une distance l'une de l'autre de $60 \mathrm{~cm}$.; la plus haute est à $20 \mathrm{~cm}$. du bout supérieur du poteau, qui est surmonté d'une tige lourde cn fer galvanisé, de la forme ordinaire. Lorsquilil y a un changement de direcion de la ligne, on a placé des appuis lourds enfoncés dans du béton pour supporter la tension en ce point. Il y aura plus de $208 \mathrm{~km}$. de lignes aériennes dans le réseau alimenté par cette entreprise.

Sur certains points de la ligne, il a été bien difficile de transporter le matériel, en particulier sur les montagnes où il n'existait même pas de sentier; aussi fut-on obligé de construire, sur quelques-unes des sections, un chemin de fer à voie étroite de $8 \mathrm{~km}$. de longueur, exploité sur les pentes les plus faciles par la traction à cheval, et là où la pente était trop forte, au moyen de trtuils à vapeur. La construction et l'entretien de cette voie sur une certaine étendue de terrain marécageux pendant une période de pluies incessantes, a été une des difficultés les plus sérieuses à surmonter par les entrepreneurs.

En plus de la force motrice à fournir aux carrières, moulins, etc., du voisinage, la station fournira l'électricité à un chemin de fer à courant triphasé dune longueur de $32 \mathrm{kms}$.

\section{(L'Indiustrie Electrique.)}

\section{Protection du bois contre les thermites.}

Tous les bois, à l'exception de quelques-uns d'un prix très élevé, sont sujets aux déprédations causées par les thermites, car jusqu'ici on n'a trouvé aucun traitement efficace pour lá protection du bois contre cette perte. La créosote et autres huiles minérales ont été essayées, mais elles ne sont pas permanentes, n'imprègnent que superficiellement le bois, et ne sont pas utulisables dans les travaux intérieurs. D'autres produits chimiques ont été employés, mais ou ils ne sont pas efficaces ou ils sont trop coûteux. Un nouveau procédé semble pourtant combattre toutes ces objections, et il consiste à bouillir d'abord le bois dans une solution de saccharine qui expulse l'air latent. Dans le refroidissement qui suit, la solution est absorbée par les tissus avec ce résultat que le bois est renforcé et amélioré comme qualité. Il est ensuite séché artificiellement par le procédé courant et, lorsque l'application est complète, le bois est complètement imprégné. Afin de préserver le bois de l'attaque des thermites, il suffit de mélanger à la saccharine un produit nocif pour ces insectes, produits qui sont absorbés par le bois en même temps que la saccharine. Le surcroit de dépense consiste simplement dans l'achat des produits ajoutés dont le prix est très peu élevé. Des échantillons ainsi traités ont été envoyés aux Indes et les rapports qui ont été reçus annoncent des résul. tats satisfaisants.

(Revue Industrielle du Centre.)

\section{La plus grande turbine du monde}

D'après la Revue de Métallurgie, la plus grande turbine du monde vient d'être installée à Shawinigan Falls, près de Québec, au Canada, par la Shawinigan Water and Power Co.

Cette turbine, qui a été construite par la Cie Morris, de Philadelphie, dans le delai de cinq mois, développe ro 500 chevaux sous une chute de 39 mèıres. Les dimensions sont de $9 \mathrm{~m}$. de haut, $6 \mathrm{~m}$. de large et $8 \mathrm{~m}$. I o de longueur d'arbre entre coussinets. Son poids est de 150 tonnes.

\section{La Houille Blanche aux Etats.Unis}

La Concord Electric Company a récemment installé une usine hydro-électrique à Sewall's Fall, dans le New-Hampshire, pour utiliser une chute, qui est de 16 pieds $\left(4^{\mathrm{m}} 88\right)$ en hautes eaux, nais qui tombe à 12 pieds $(3 \mathrm{~m} 66)$ pendant la période des basses eaux. C’est donc une très basse chute. Pour le moment, l'installation ne comporte que deux turbines de 700 chevaux, actionnant chacune un alternateur de 500 kilowatts, tournant à sootours par minute, et ploduisant du courant triphasé à 60 périodes sous 2600 volts. Les turbines sont à axe vertical et du type Francis centripète parallèle; elles se composent de 3 couronnes mobiles en bronze disposées les unes au-dessus des autres, et de 3 distributeurs. Les 3 couronnes sont semblables, et leur diametre est de $1^{\mathrm{m}_{4}} 4$.
A Trenton-Falls, dans l'Etat de New-Yurk, la Utica Gas and Electric Company a installé une usine hydro-électrique sur le West Canada Cieek. La chute est ici de 80 mètres et elle peut produire 16000 chevaux.

La prise d'eau est constituée par un barrage qui a $18 \mathrm{~m} 30$ de hauteur et 92 mètres de longueur. Il est construit en béton et est implanté suivant un arc de cercle de $244 \mathrm{~m}$. de rayon. Le barrage est muni de deux déversoirs. L'un d'eux, long de $30^{m} 50$, est établi parallèlement à la rivière, à om61 au-dessous de la crête du barrage; lautre, suivant une méthode fort en usage en Amérique, est établi au milieu du barrage lui-même sur une longueur de $49 \mathrm{~m}$, et à $\mathrm{I}^{\mathrm{m}} 25$ en dessous de la crête.

La conduite d'amenée a $2^{\mathrm{m}}$ ro de diamètre et prend l'eau dans le réservoir au moyen de deux tuyaux de $\mathrm{I}^{\mathrm{m}} 54$ de diamètre, qui se raccordent avec la conduite générale au moyen d'une tubulure en forme d'Y. Sur $885 \mathrm{~m}$. la.conduite est constituée par des douves de bois de sapin maintenues par des feuillards, et sur 246 autres mètres elle est en tôle d'acier.

L'installation comporte actuellement 6 turbines verticales, dont 4 pour les alternateurs et 2 pour les excitatrices Les grosses turbines sont du type centrituge de Fourneyron; elles tournent à 30 tours et actionnent directement six alternateurs de 2000 chevaux produisant du courant triphasé à 60 périodes sous 2300 volts. Les turbines des excitatrices sont du type Girard. Le courant produit par les alternateurs est envoyé dans des transformateurs qui élèvent sa tension à 23 ovo volts. De là il se rend à Utıca, c'est-à-dire à une distance de $23 \mathrm{kms}$.

En utilisant dautres chutes existant dans les environs, la Company se propose de produire 23000 chevaux.

\section{BIBLIOGIATHIE}

Etude expérimentale du Ciment armé, par R. Feret, encyclopedie Léchalas - Paris, Gauthier-Villard.

Quand on se prépare à couper les feullets de ce volume, on pense tout naturellement qu'on va y trouver tout d'abord, comme suite aux procédés opératoires élémentaires, une théorı de la résistance des structures en béton de ciment armé; on n'est pas déçu, et les deux premières parties du livre, environ la bonne moitıe de son texte, sont pour tradurre la manière dont $\mathrm{M}$. Feret comprend la théorie, à l'heure actuelle. Nous avons apprécıé la manière méthodique dont l'auteur expose cette théorie, et surtout le secours qu'll sait trouver dans les constructions graphıques pour évaluer des quantités que le calcul, tant algébrique que numérique, ne saurait donner sans complications prohıbituves.

La trosieme partie, grosse de 150 pages, est celle que nous considérons et de beaucoup comme la plus curieuse. Ce n'est pas pour rabaisser le reste ! C'est celle où $M$. Feret nous donne la bibliographie du béton de ciment arme. - C'est formidable! et encore que certainement chacun de ceux qui la parcourront puisse y relever des lacunes, car les applications dans les dix dernières années ont été prodigieusement nombreuses, c'est suggestif au premier chef et... encourageant Après nous avoir en quelque sol te dit dans ses deux premières parties: "Vollà comment on peut concevolr la maniète dont se comporte le béton de ciment armé! M Feret semble nous dire: "Maintenant vollà le bien et le mal qu'on en a dit, avec la constatation j'une grande partie de ce qu'on en a fait. Jugez vousmêmes!n

Dans la quatrième partie, l'auteur, qui est une des autorités en la matière nous dit: "Quant à moi, voici dans quel sens je cherche, "voulez-vous me suivre? " Et cela encore est remarquable.

Nous avons letrouvé dans cette partie de l'ouvrage les expériences si connues de l'auteur sur la compacité, les sables normaux, etc., si utiles au point de vue des prévisions qu'on en peut inférer quant aux travaux qu'on doit entreprendre. Les recherches sur l'adhérence des pâtes et des armatures, etc., se résumant en conseils pratiques.

Maintenant analyserons-nous l'ouvrage dans son détail ? Non! il y

a des choses qu'on ne fait pas à demi et nous n'avons pas la place! Ce que nous pouvons et devons faire, en toute conscience, c'est de dirc aux ingénieurs: achctez-le, ses 800 pages vous dispenseront d'en lire plusieurs milliers et vous inciteront à réfléchir.

Méthodes économiques de combustion dans les chaudières à vapeur, par J. Izan I, Ingénieur Civil des Mines. - Dunod et Pinal, 49, quai des Grands-Augustins, Paris VIe.

M. Izart n'est plus un inconnu pour nos lecteurs ; en février 1905 , nous avons analysé pour eux sa très intéressante plaquette: Les méthodes modernes de paiement des salaires, et en septembre de la même année, un autre opuscule: L'Economie dans lat chaufferie. - 
Il nous donne aujourdhui sur ce dernier sujet les éclaircissements qu'il avait dû négliger alors. Il analyse la combustion, ce qu'elle est, l'air qu'll lu filut, la chaleur qui en résulte, les pertes auxquelles l'industriel doit savoir tarre leur part: il nous gunde dans le chorx si délicat du combustible optimum selon les cas, dans son achat, son essa1, sa conservation. 11 nous apprend comment 1 faut s'en servir et par quels appareils et quels procédes nous saurons toujours où nous en sommes et ce que nous faisons.

Tout cela, M. Izart, nous l'expose simplement, bonnement, sans algèbre rébarbative: des diagrammes, des tableaux clairs, parfo s (bien rarement), une équation simple, pas même, une proportion, une règle de trois ' - Aussi on a plaisir à le lire: lil est de l'Ecole de Puinsot qui dans son mémoire sur la rotation des corps disait :

"Ce n'est point dans le calcul (algébrique) que résıde cet art qui " nous tait découvrir, mais dans cette considération attentive des " choses, où l'esprit cherche avant tout à s'en faire une idée, en " essavant par l'analyse proprement dite, de les décomposer en " d'autres plus simplıs, atin de les ravoir ensuite, comme si elles " étatent formées par la réunion de ces choses simples dont il a une " pleme connaissance Ce n'est pas que les choses solent composées " Je cette manière, mais c'est notre seule mantère de les voir, de " nous en faire une idée, et partant de les connaître".

Le livre de M. Izart est présenté dans cet esprit: nous ne voyons pas meilleur éloge à en faire, et nous n'hesitons pas à en conseiller l'usage à tous nos confrères. - Tous les jours la houille noire est anpelce à être l'auxilıaire de la houlle blanche, et réciproquement, et il faut savorr les trater l'une et l'autre comme elles le meritent.

Commandant Audebrand. Ingéneur,

Ancien élève de l'Ĺcole polytechnique.

L'électricité industrielle mise à la portée de l'ouvrier, par E. Rosenbarg, traduit de l'allemand par A. Mauduit, ancien élève de l'Ecole polytechnique, $2^{e}$ édition. In-8 de $x-490$ pages, avec 312 fig. Dinod et Pinat, éditeurs, Paris.

Cet ouvrage, dû à la plume d'un ingénieur allemand très connu des spécialistes par ses publıcations techniques, a obtenu un grand succès en Allemagne et a été traduit en anglais. M. Mauduit a rendu service au public français en le lu faisant connaitre par une traduction dont l'épuisement rapide a nécessité une seconde édition. Ce travall, d'un caractère foncièrement pratique, est particulièrement destıné à louvrıer qui, ayant à manipuler les machınes électriques si diverses utilisées actuellement dans l'ındustrie, veut en comprendre le fonctionnement et se rendre capable de parer aux dérangements qui s'y produsent.

Mais, par suite de son caractère élémentaire, il convient à ceuy qui veulent se familiariser avec l'Electricité industrılle, quel que solt le niveau de leur instruction; et comme, malgré sa simplicité, il pénètre profondément dans l'étude de l'électricité, décrit et explique le fonctionnement des appareils les plus divers, 11 iendra service aux chefs et employés d'usincs ou de maisons de commerce utilisant des installations électriques, aux élèves des écoles professionnelles, et a tous ccux qui s'intéressent aux machines électriques et veulent en connaître les propriétés et la conduite.

Fdisons remarquer le véritable tour de force accomplı par l'auteur dans les chapitres : lesMachines à courants alternatifs simples et volyphases, expliquées clarrement avec les seules connarssances de l'Ecole primare.

La rapidité avec laquelle se sont enlevés les deux mille exemplaires de la première édition francaise montre surabondamment que le public a compris et apprécié le but de l'ouvrage: aussı M. Mauduit a-t-1l cru devoir profiter de la seconde édition pour ajouter un complément de grande actualité.

Presque toutes lés grandes villes et même, dans certaines contrées, les moindres villages, sont éclairés mantenant à l'electricité; le traducteur a donc pensé être utile en exposant les différents systèmes de distrıbution de l'énergie électrıque, le fonctıonnement des compteurs et les principes élementarres de la photométrie, c'est-àdire la comparatson, au point de vue des résuliats économiques, des differentes sources lumineuses, et en particulier de celles qui font l'objet des plus récentes inventions.

Mise en valeur des moyennes et basses chutes d'eau en France, ouvrage publié sous les auspices de M. le Ministre de l'Agriculture, par Henri Bresson, avec préface de M. MAX DE $\mathrm{N}$ insouty, un volume in-8 de xxI-278 pages, avec 126 figures et une planche hors texte. Frix : 7 fr. 50. H. Dunod er E. Pinat, éditeurs, Paris.

Tout le monde connaît aujourd'hui la houille blanche, ct l'on sait combien l'utilisation des grandes chutes d'eau en pays de montagne a contribué aux progrès de nombre d'industries.

En dehors des régions montagneuses, il existe, dans presque toute la France, quantité de cours d'eau susceptıbles d'être aménagés par de petits barrages en vue de produire de l'énergie électrique, et bien que chacun ne présente, en général, qu'une assez fauble puissance, leur ensemble ne constitue pas moins un élement de richesse important, et dont la mise en valeur est relativement facile.

M. Henri Bresson, après avoir, l'un des premiers de sa contrée, tiré personnellement partıe d'un barrage longtemps inutilısé pour la production de la lumière et de la force motrice à ses besoins personnels, s'est fait l'arient propagandiste de l'emploi de l'énergie des cours C'eau de plaine, ce dont nous le félıcitons grandement. La houille blanche n'est pas un apanage exclusif de la montagne et elle est aussi bien unlisable dans les planes lorsqu'on sait s'en servir.

Dans l'ouvrage qu'il présente aujourd'hui au public, M. H. Bresson montre avec quel empressement l'exemple qu'il a donné a été suivi dans la régıon normande, voisine de sa propre installation. Toutefois, il ne s'agit pas ici d'une elude purement régionale; la portée de ce Iivre est plus haute. C'est, avant tout, une cuvre de vulgarisation qui s'adresse principalement aux riverains des cours d'eau e:, en particulier, aux nombreux propriétaires des barrages inutilisés. Les exemples remarquables que donne $M$. H. Bresson devraient suffie pour convaincre ceux qui sont restés jusqu'ici refractaires au progrès des nombreux avantage qu'ils pourraient aisément retirer de la mise en valeur rationnelle de lı source d'énergie gratuite et inépuique la nature a mise à leur dispositıon.

Règles normales de l'Association des électriciens allemands pour la comparaison et l'essai des machines et transformateurs électriques, suivies de commentaires, par $M$. G. Detrmar, traduit de l'allemand par F. Loppé et A. Thouvenot, ingénieurs. In-8 de 72 pages. Prix: 2 tr. 50. Dunod et Pinat, édi. teurs, Paris.

M M. Loppé et Thouvenot ont eu l'heureuse idée de traduire les rèules normales de la Verband deutscher Electrotechniker pour la comparaison et l'essai des machines électriques et des transformateurs.

Ce travail d'un haut intérêt concerne la puissance, l'élévation de température, les surcharges, l'isolement, le rendement, les méthodes pour déterminer ce rendement, la variation de tension, etc. Il est suvi des intéressants commentaires de $M$. Dettmar et précédé de définitions des plus utiles.

Les tremblements de terre, leur origine possible, les tremblements de terre au Pérou, par Emile GUarini, professeur à l'Ecole d'arts et métiers de Lima. In-8 de 26 pages, 2 fr. Dinod et Pinat, éditeurs, Paris.

Dans cette brochure, l'auteur rappelle les plus importantes periurbations sismiques du globe, réunit et discute les observations et les phénomènes qui précèdent ou suivent les tremblements de terre, expose les diverses théories émises pour expliquer l'origine de ces phenomènes.

$M$. Guarini conclut que l'hypothèse électrique permet seule d'expliquer que la côte du Pérou est plus ravagee que beaucoup d'autres régions.

La théorie électrique s'accorde en outre avec certrins phénomènes constatés; et si le tremblement de terre était toujours d'origine électrique, il serait peut-être possıble de l'éviter. La thèse de M. Guarini, ne manque pas d'originalité, et on la lira avec d'autant plus d'intérêt que les terribles catastrophes de San-Francisco et de Vdlparaiso sont encore présentes à la mémoire de tous.

\section{LIVRES NOUVEAUX EN FRANCE ET A L'ÉTRANGER}

Die Gruppenwasserwerke in der Provinz Reinhessen, Bовнавr. $3 \mathrm{fr} .75$.

French Abreviations, commercixl, financial and general. E. LaTham, 3 fr.

Résistance des matériaux appliquée aux constructions, méthodes pratiques par le calcul et la statique graphique. Aragos, I $5 \mathrm{fr}$.

Ueber Wasserkraft und Wasserversorgungsanlagen. ScHLotTHAUER, 8 fr. 75 .

Projectierung von Elecktrizstatswerken. F. Hoppe, 4 fr. $7^{5}$. Wissenwertes aus dem Dynamobau fur installateure. ScHuzz, 3 fr. 25.

Lichtstrahlung und Beleuchtung. HoGN $\mathrm{R}, 3$ fr. $7^{5}$.

L'Imprimeur-Gérant: P. LEGENDRE. 